\title{
Optimization Model of Balanced Resource Allocation in Construction Project Group
}

\author{
CHANG Chunguang ${ }^{1, a^{*}}$, QI Minghao ${ }^{2, b}$, SONG Ziwei ${ }^{3, c}$ \\ ${ }^{1}$ School of Management, Shenyang Jianzhu University, Shenyang 110168, China \\ ${ }^{2}$ China Construction Eighth Engineering Division. CROP.LTD Tianjin Branch, Tianjin 300452, China \\ ${ }^{3}$ School of Public Management, Beijing City University, Beijing 100000 \\ achang chunguang@163.com, ${ }^{b}$ 360685487@qq.com, ccg7788@sohu.com
}

Keywords: Optimization model; Balanced resource allocation; Construction project group

\begin{abstract}
In construction project group, the construction process is numerous, the resources required are multifarious, the resources allocation is difficult, and it is easy to appear resource allocation imbalance within a certain period of time. To implement the balance allocation of resources among varied projects, the optimization allocation model of resource equilibrium in project group is established. In constraints, construction process in each project should obey logic order; supply quantity of each resource per day should be within its lower limit and upper limit scope. A heuristic algorithm is proposed to solve above model so as to realize the balanced allocation of resources. Through the analysis of examples in application, its validity is validated, and it will provide reference for practical resource imbalance problems in engineering project group.
\end{abstract}

\section{Introduction}

Resource consumption quantity and categories are different during varied stages of the construction process in project groups. Resource consumption is very imbalance on varied stages. It very difficult for management, and construction cost is increased. Resource allocation should be adjusted so as to realize balanced resource consumption and avoid large fluctuating on resource consumption.

A robust genetic algorithm for resource constrained project scheduling problem is presented in [1]. An active resource allocation method that the level of actual resource mobilization can be changed by the construction period is demonstrated in [2]. A resource allocation model for project scheduling is proposed in [3]. To solve resource allocation project scheduling problem and resource leveling problem at the same time, two new metaheuristic algorithms are utilized in [4]. A class of resource leveling optimization problems often met in modern project management is studied in [5]. For multi-resource allocation balance in construction project group, how to establish effective optimal model is worth researching in more profoundly.

\section{Optimization Model of Balanced Resource Consumption in Project Group}

Variables Explanation. Variables are described as follows.

$m, n$ - number of engineering projects in project groups, production processes in each project.

$(i, j)$-production process $j$ in project $i$.

$r$-number of resource category on each production process.

$u_{i j k}(t)$ - usage amount of resource $k$ on day $t$ on production process $(i, j)$.

$Q_{k}(t), \bar{Q}_{k}$-total usage amount of resource $k$ on day $t$, average usage amount of resource $k$ during total completion time of project group.

$\omega_{k}(t), \omega_{k}^{\prime}(t)$-lower limit, upper limit of usage amount of resource $k$ on day $t$.

$T_{i}, T$-completion time of project $i$, total completion time of project group.

$t_{i j}, \Delta t_{i j}$-standard time of production process $j$ in project $i$, free float time of production process $(i, j)$.

$\sigma^{2}$ - total variance of resource usage amount for project group. 
$b_{i j}$-the earliest begin time of production process $j$ in project $i$.

$P_{i j}$-preceding activity set of production process $j$ in project $i$.

Basic analysis and Modeling Establishing. For $(i, j)$, once the completion time of one project is changed, a series of chain reactions will be generated, conflict between various projects will be led. It is not easy to be adjusted, and time is delayed so as to delay the completion time of project group. To make the usage amount of each resource more balanced, variance is needed to be as a measure index. And optimization model of balanced resource consumption in project group is established as follows.

$$
\begin{gathered}
\min \sigma^{2}=\frac{1}{T} \sum_{t=1}^{T} \sum_{k=1}^{r}\left[Q_{k}(t)-\overline{Q_{k}}\right]^{2} \\
\text { s.t. } \quad b_{i j}-b_{i h} \geq t_{i h} \quad \forall h \in P_{i j} i=1,2, \mathrm{~L}, m ; j=1,2, \mathrm{~L}, n \\
\omega_{k}(t) \leq Q_{k}(t) \quad k=1,2, \mathrm{~L}, r ; t=1,2, \mathrm{~L} T \\
Q_{k}(t) \leq \omega_{k}^{\prime}(t) \quad k=1,2, \mathrm{~L}, r ; t=1,2, \mathrm{~L} T \\
u_{i j k}(t) \geq 0 \quad i=1,2, \mathrm{~L}, m ; j=1,2, \mathrm{~L}, n ; k=1,2, \mathrm{~L}, r ; t=1,2, \mathrm{~L} T
\end{gathered}
$$

Where, $Q_{k}(t)=\sum_{i=1}^{m} \sum_{j=1}^{n} u_{i j k}(t), T=\max \left\{T_{i}\right\}, \bar{Q}_{k}=\frac{1}{T} \sum_{t=1}^{T} \sum_{i=1}^{m} \sum_{j=1}^{n} u_{i j k}(t)$. Formula (1) indicates that the smaller is the variance, the better is the scheme. Formula (2) indicates that the order of production processes in each project should meet process sequence requirements. Formula (3) indicates that the usage amount of resource $k$ on day $t$ is not less than lower limit of usage amount. Formula (4) indicates that the usage amount of resource $k$ on day $t$ is not more than upper limit of usage amount. Formula (5) indicates that variables should be nonnegative number.

\section{Model Analysis and Heuristic Algorithm}

Model analysis and solving idea. In formula (1), $\frac{1}{T} \sum_{t=1}^{T} \sum_{k=1}^{r}\left[Q_{k}(t)-\overline{Q_{k}}\right]^{2}=\frac{1}{T} \sum_{t=1}^{T} \sum_{k=1}^{r} Q_{k}^{2}(t)-\sum_{k=1}^{r}{\overline{Q_{k}}}^{2}$. Considering $\overline{Q_{k}}$ is constant, $\sum_{t=1}^{T} \sum_{k=1}^{r} Q_{k}^{2}(t)=\sum_{t=1}^{T}\left\{\left[Q_{1}(t)\right]^{2}+\left[Q_{2}(t)\right]^{2}+\cdots+\left[Q_{r}(t)\right]^{2}\right.$.

For any non-critical activity $(g, h)$, standard time of $(g, h)$ is $t_{g h}$. Free Float time of $(g, h)$ is $\Delta t_{g h}$. According to the initial resource allocation scheme in plan network diagram, the production processes are arranged by the earliest begin time. Set begin time of the production process as $t$, if the begin time of the production process is postponed one day, the resource usage amount is reduced as $\sum_{k=1}^{r} u_{g h k}(t)$ on day $t$. However, the resource usage amount is increased as $\sum_{k=1}^{r} u_{g h k}(t)$ on day $t+t_{g h}+1$. The time adjust scope of production process should be within the scope of Free Float time. On above analysis, the alteration amount of $\sigma^{2}$ can be calculated as formula (6).

$$
\begin{gathered}
\Delta \sigma^{2}=\sum_{k=1}^{r}\left[\sum_{i=1}^{m} \sum_{j=1}^{n} u_{i j k}(t)\right]^{2}+\sum_{k=1}^{r}\left[\sum_{i=1}^{m} \sum_{j=1}^{n} u_{i j k}\left(t+t_{g h}+1\right)\right]^{2}-\sum_{k=1}^{r}\left[\sum_{i=1}^{m} \sum_{j=1}^{n} u_{i j k}(t)-u_{g h k}(t)\right]^{2}-\sum_{k=1}^{r}\left[\sum_{i=1}^{m} \sum_{j=1}^{n} u_{i j k}\left(t+t_{g h}+1\right)-u_{g h k}(t)\right]^{2} \\
=2 \cdot \sum_{k=1}^{r}\left\{u_{g h k}(t) \cdot\left[Q_{k}(t)-Q_{k}\left(t+t_{g h}+1\right)\right]-\left[u_{g h k}(t)\right]^{2}\right\}
\end{gathered}
$$

By formula (6), if the value of $\Delta \sigma^{2} \geq 0$, the adjusted resource allocation scheme is more reasonable on resource equilibrium by postponed one day begin time than initial one. By above method, postpone begin time of non-critical activity one by one until $\Delta \sigma^{2}<0$.

Heuristic Algorithm Designing. Heuristic algorithm for above model is designed as follows.

Step 1: Draw the activity-on-arrow network of each single project. 
Step 2: Connect the activity-on-arrow network of each single project by adding a common start node and a common terminal node, integrate single project into one large project.

Step 3: Calculate parameter values of the activity-on-arrow network, find out non-critical activities on each project, distinguish the constructing time sequence of above non-critical activities.

Step 4: Adjust non-critical activities one by one by formula (2) and formula (6). The adjustment sequence is based on the ascending order of distance between the non-critical activity to the last node.

Step 5: Judge whether the adjustment scheme meets formula (3) and formula (4), if it does not meet formula (3) and formula (4), the adjustment is cancelled.

Step 6: If the non-critical activities are not all adjusted, go to step 3, else the algorithm ends.

\section{Instance Application}

The project group consists of 3 projects. The resource allocation scheme of each project is arranged preliminarily as figure 1 . Where, production process $(1,3)$ and $(1,4)$ in project 1 are dummy activities . $t_{i j}\left(u_{i j 1}, u_{i j 2}, \ldots u_{i j k}\right)$ denotes that the construction time of $(i, j)$ is $t_{i j}$, the resource usage amount are respectively $u_{i j 1}, u_{i j 2}, \ldots u_{i j k}$. Here, $\omega_{k}(t)=5, \omega_{k}^{\prime}(t)=50$. By program evaluation and review technic (PERT), the critical path is marked by thick lines in figure 1.

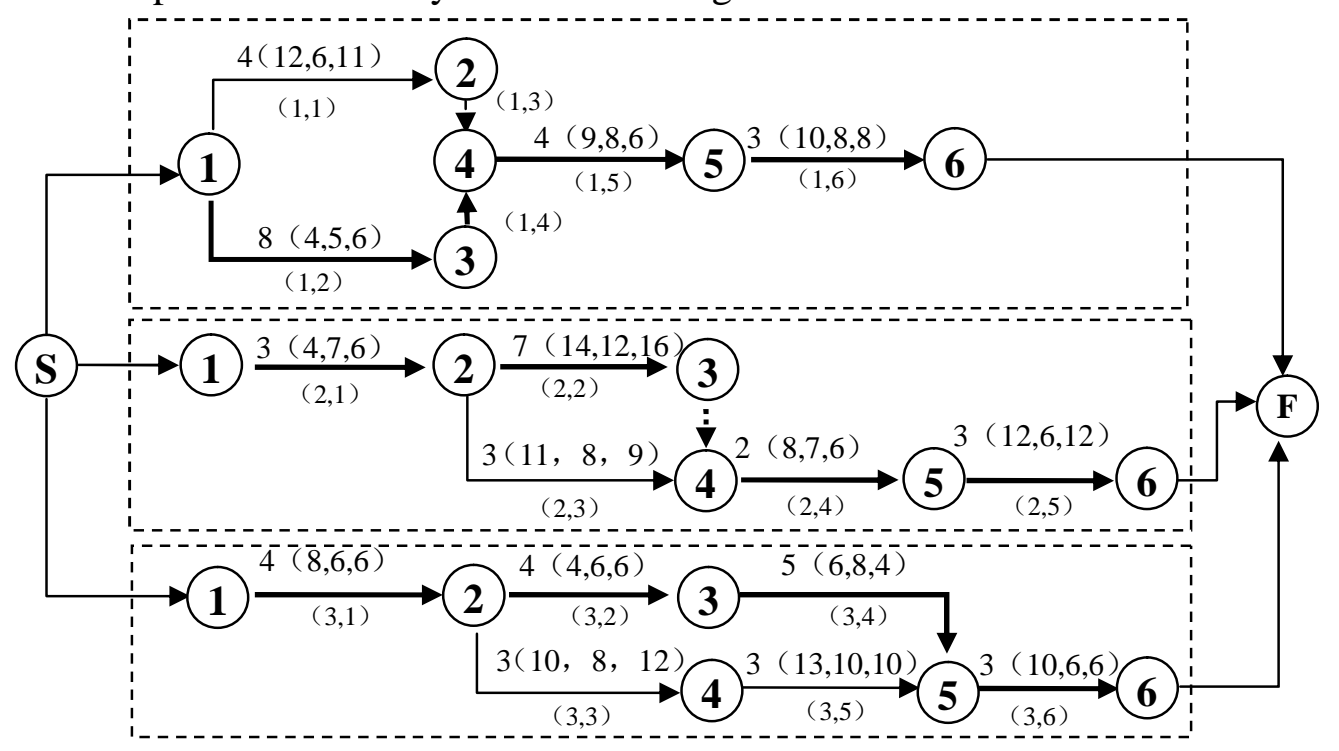

Figure 1 Plan network diagram with resource allocation

In non-critical activity $(1,1),(2,3),(3,3)$ and $(3,5)$, production process $(3,3)$ must be performed directly after $(3,1)$ without postponing according to the process requirement. Thus, adjustment of production process $(1,1),(2,3)$ and $(3,3)$ is discussed here. Non-critical activity $(3,5)$ should be adjusted firstly by above discussion, the begin time of $(3,5)$ is the $8^{\text {th }}$ day, the production process needs 3 days to be completed. Free Float of $(3,5)$ is 3 days. Corresponding usage amounts of varied resources of $(3,5)$ is $\left(u_{351}, u_{352}, u_{353}\right)=(13,10,10)$. The total resource usage amount of the $8^{\text {th }}$ day on $(3,5)$ is $\left[Q_{1}(8), Q_{2}(8), Q_{3}(8)\right]=(35,33,38)$. If 1 day is postponed, the resource usage amount on the $11^{\text {th }}$ day $\left[Q_{1}(11), Q_{2}(11), Q_{3}(11)\right]=(23,23,16)$. According to formula (6), if $\Delta \sigma^{2}<0,(3,5)$ can not be postponed; otherwise, $\Delta \sigma^{2} \geq 0,(3,5)$ can be postponed 1 day. Then the result by postponing 1 day needs to be calculated, and the plan network diagram with resource allocation should be adjusted. On the adjusted plan network diagram with resource allocation, 1 more day is postponed on non-critical activity $(3,5)$, and repeated above steps. The detail calculated result is listed in table 1.

By table 1, $(3,5)$ should be postponed 3 days to improve the balance degree of varied resources allocation. In the same way, for $(2,3)$ and $(1,1)$, non-critical activity $(2,3)$ is adjusted firstly according above adjustment rule, and $(1,1)$ is adjusted finally. The allocation calculation for resource balance for $(2,3)$ and $(1,1)$ is listed in table 1 . $(2,3)$ should be postponed 4 days, $(1,1)$ should not be postponed. 
Table 1 allocation calculation for resource balance for non-critical activity

\begin{tabular}{|c|c|c|c|c|c|c|c|}
\hline $\begin{array}{l}\text { Production } \\
\text { process }\end{array}$ & $\begin{array}{l}\text { Postponed } \\
\text { days }\end{array}$ & $\begin{array}{c}\text { Usage amounts of varied } \\
\text { resources }\end{array}$ & $\Delta \sigma^{2}$ & $\begin{array}{c}\text { Production } \\
\text { process }\end{array}$ & $\begin{array}{l}\text { Postponed } \\
\text { days }\end{array}$ & $\begin{array}{l}\text { Usage amounts of } \\
\text { varied resources }\end{array}$ & $\Delta \sigma^{2}$ \\
\hline \multirow{3}{*}{$(3,5)$} & 1 & \multirow{3}{*}{$\left(u_{351}, u_{352}, u_{353}\right)=(13,10,10)$} & $214>0$ & \multirow{4}{*}{$(2,3)$} & 1 & \multirow{4}{*}{$\begin{array}{l}\left(u_{231}, u_{232}, u_{233}\right) \\
\quad=(11,8,9)\end{array}$} & $82>0$ \\
\hline & 2 & & $456>0$ & & 2 & & $564>0$ \\
\hline & 3 & & $186>0$ & & 3 & & $266>0$ \\
\hline$(1,1)$ & 1 & $\left(u_{111}, u_{112}, u_{113}\right)=(12,6,11)$ & $-1024<0$ & & 4 & & $266>0$ \\
\hline
\end{tabular}

$T=\max \left\{T_{1}, T_{2}, T_{3}\right\}=16, \overline{Q_{1}}=29.7, \overline{Q_{2}}=27.6, \overline{Q_{3}}=31.1$. In initial plan scheme, $\sigma^{2}=3088.375$ $-29.7^{2}-27.6^{2}-31.1^{2}=477.315$. In above optimized plan scheme, $\sigma^{2}=2845.375-29.7^{2}-27.6^{2}$ $-31.1^{2}=234.315, \sigma^{2}$ is reduced $50.91 \%$. The adjustment scheme meets the requirement of process, resource usage amount lower limit and upper limit. The improve result of adjustment scheme is obviously, and it benefits for allocating resources in more reasonable way.

\section{Conclusions}

How to obtain balanced resource allocation is a key problem in construction project group. The optimization objective of balanced resource allocation is to improve resource usage efficient, benefit for management, prevent resource requirement collision. By optimization model, under the condition of completion time of each project, resources can be allocated in more balance way among varied projects, and the construction cost can be reduced. In project group domain, advanced modeling and optimization technique for resource allocation is the main research direction in the future.

\section{Acknowledgements}

This work was supported by Humanities and Social Sciences Project from Ministry of Education of the People's Republic of China(15YJA630001); The Natural Science Foundation of Liaoning Province (201502794); Project from Ministry of Housing and Urban-Rural Development of the People's Republic of China(2014-R3-014); Science Research Program of Liaoning Province Education Administration: Liaoning city public safety management by E-CBR (W2014084) \& Research on the industry-university-research-user cooperative innovation mechanism of the market oriented research in Liaoning (W2014085); Work Safety Project of State Administration (2013-13) and Shenyang Scientific and Technological Planning (F15-198-5-15).

\section{References}

[1] J. Alcaraz, C. Maroto. A Robust Genetic Algorithm for Resource Allocation in Project Scheduling. Annals of Operations Research,102(2001) 83-109.

[2] Leen-Seok Kang,Hyoun-Seok Moon,Chang-Hoon Min,Sung-Keun Kim,Hyeon Seung Kim. Developing an active resource allocation algorithm considering resource supply and demand in a construction site. KSCE Journal of Civil Engineering,19(2015,) 17-27.

[3] Wolfram Wiesemann, Daniel Kuhn, Berç Rustem. Multi-resource allocation in stochastic project scheduling. Annals of Operations Research, 193(2012)193-220.

[4] M. Khanzadi,A. Kaveh, M. Alipour,H. Karimi Aghmiuni. Application of CBO and CSS for Resource Allocation and Resource Leveling Problem. Iranian Journal of Science and Technology, Transactions of Civil Engineering, 40(2016)1-10.

[5] Christos Kyriklidis,Vassilios Vassiliadis, Konstantinos Kirytopoulos,Georgios Dounias. Hybrid nature-inspired intelligence for the resource leveling problem. Operational Research, 14(2014) 387-407. 